\title{
A Glance at Rastin Banking
}

\author{
Bijan Bidabad \\ B.A., M.Sc., Ph.D., Post-Doc. \\ Professor \\ Economics and Chief Islamic Banking Advisor \\ Bank Melli, Iran \\ E-mail:bijan@bidabad.com
}

\begin{abstract}
To design an operational Islamic banking system, we tried to mix theoretical and experimental knowledge to develop Rastin Banking. Rastin Banking is a completely new solution to banking based on Islamic and ethical teachings with the scientific and technological approach. Some parts and modules of Rastin Banking have been implemented in Bank Melli Iran. The installed parts of the system had well-functioning and attracted depositors and investors, and since the procedures and instructions are well defined, the bank's staff executed its procedures easily. The results of the test system were very satisfactory and more than expectation. Rastin Banking is a nationwide project with lots of subtle technical points. This system is an open-source banking model, and all banks around the globe can easily install and use it. We wish Rastin Banking could put important steps to remove Riba and establish Islamic banking throughout the globe.
\end{abstract}

\section{Keywords: Rastin Banking, Rastin PLS banking, Islamic banking, Interest-free banking}

\section{Introduction}

Many economists around the world have done their best to eliminate Riba from banking activities, but have not achieved much. In this direction, Rastin Banking, in compliance with Sharia commands, has been compiled not only to eliminate Riba but also to institutionalize various teachings of justice and Islamic ethics in banking activities. Good points of Rastin Banking in all fields of banking, financial, economic, ethical, social, and international activities are so expanded that it can be regarded as a base to improve banking structure in any country.

Designing financial systems are principally complex, and when combined with ideological subjects, the complications increase. Usually, parts, internal and external processes, and subsystems of this kind of systems are facing various problems that cannot be covered easily. By increase of complexity, management of the system needs complex and special tools and since the subject of the system is financial issues, and pecuniary matters are beloved by people, by considering the most pessimistic conditions, all probable types of failures to fulfillment of obligations should be recognized and predicted in advance to reduce all cases of dishonest behaviors. Because of this issue, even well-equipped rich banks throughout the world, in spite of knowing Islamic banking advantages, have not been able to implement the task fully; or changed their minds and returned back to interest-based banking to escape the complexities of real Islamic banking and went back to prefixed interest rate banking processes, instead of profit and loss sharing.

Designing true Islamic banking needs comprehensive knowledge of ideological (Fiqh, Sharia principles, commandments and logic), philosophic (individual rights and social justice concepts), economic (individuals, firms and market behaviors), financial (accounting, auditing, financial engineering), banking (project assessment and supervision, credit operation, transactions, investment and risk), legal (laws and regulations, contracts, legal and arbitration procedures), informatics (computer systems, networks, communications, programming), organization (pillars and institutions), management (administration, control, supervision, assessment), mathematics (applied mathematics, financial mathematics, mathematical modeling), financial instruments (prevailing financial papers and securities in financial markets), business and trade (business administration, marketing, international trade), capital market (capital market structure, laws and regulations), insurance (commercial, responsibility, assets, and accident 
insurances), standards (commodity and production) issues; and in addition, should be aware of the sociology and individual and social psychologies and their corresponding problems and many other various issues.

Regarding the above subjects, Rastin Banking System was designed in Bank Malli Iran. The designer team of Rastin Banking tried to consider the processes, procedures, rules, and organization of the system comprehensively and after fifteen years of continuous work designed a total solution for Islamic banking which is unique and distinguished at international level. Rastin Banking system has all banking operations in itself. Establishment of the first profit and loss sharing branch (Ghoba) in Nov. 2010 clarified the defects of the system and new financial subsystems and complementary systems were designed and connected to Rastin Banking system because of derived operational feedbacks. Development of Rastin Banking came to a phase that other banks were interested in applying it to their own banks. Therefore, the "Compiling Committee of Operational Rules and Regulations of Rastin Banking" was established to compile the legal draft bills.

Rastin Banking is new Islamic banking, which has studied the theoretical and operational difficulties of the banking system of the country and offer legal and operational solutions on the basis of the latest scientific and operational achievements. Each subsystem, instrument, innovation, and operational procedure of Rastin Banking has been designed to remove an especial difficulty and satisfy markets and peoples' needs.

Rastin Banking fulfills all ethic definitions ever defined for ethic finance and even more. It holds the virtues of justice, charity, and generosity regarding dispositions to act in ways that benefit both the person possessing them and the person's society.I Hence, Rastin Banking benefits both the financial institution that adopts it and broader society. In Rastin Banking, humans are bound, from the knowledge of their duty as rational beings, to obey the categorical imperative to respect other rational beings. 2 This is extended in Rastin Banking that everybody had specific duties in relation to his role and endowed ability for financial intermediation. Moreover, in Rastin Banking, based on utilitarianism 3 that asserts the guiding principle of conduct should be the greatest happiness or benefit of the greatest number is observable. In this context, Rastin Banking has been developed to ensure the facilitation of benefit for the largest number of people, while not harmful to anyone. Rastin Banking fulfills Islamic principles of ethics, wherein individuals and bank are duty bound to be accountable for each and every activity conducted by them and to do right in the context of God's laws.4

To increase the compliance of banking system with principles and regulations of Islamic religion, and better access to safe financial activity and helping the economy to bloom, fair distribution of possibilities and opportunities, job creation and increasing the welfare of the society, the Rastin Banking System was designed and all banks can carry out their activities on this basis.

Rastin Banking System is based on special operational, financial, economic, ethical, social, legal, international and organizational principles that based upon the latest scientific achievements of humankind in the field of science and technology with the aim of growth and development of the economy and banking of the society. For example:

In order to facilitate legal activities of Rastin Banking, some improvements have been carried out on auxiliary Islamic contracts. To fulfill the necessary legislative needs of bank sharing activities, joint investment funds and facilitating social insurance activities, some new legal institutions are also defined as "Fund with variable capital". To distinguish profit from Riba, certain measures were also defined.

In order to prevent squandering (Israf) and to reform banking sources consumptions, which have worse economic and social consequences than Riba, and to increase efficiency, the bank is obliged to follow specific regulations concerning its activities and finance only those projects, which have observed certain considerations and criteria in their project proposal.

Bank can finance projects of restoring uncultivated lands by supporting qualified applicants for the promotion of employment and development of agriculture, industry, mining, housing, and tourism in the development of different regions of the country.

I Definitions from the Oxford Dictionary

2 Kant definition

3 Utilitarianism approach

4 Accounting and Auditing Organization for Islamic Financial Institutions (GSIFI) Governance Standard No. 7: Corporate Social Responsibility Conduct and Disclosure for Islamic Financial Institutions. 
Bank and parties involved in Rastin Banking contracts should observe the supervisory compiled regulations concerning financial transparency, information disclosure, and corporate governance. All contracts with the bank are considered as official documents and enforceable. These contracts will be carried out by a unit of execution of enforceable documents in the bank.

A collection of Rastin Banking approaches is defined in Rastin Banking Bill and Operational Bylaw of Rastin Banking. Regulations of this banking system and all developments, improvements and designing its subsystems and new processes or concordance new subsystems with prevailing processes should be carried out by considering the content of the cited Bill and Bylaw. In addition, all future supplements and operational workflows should be designed in a way that not to contain fixed interest (Riba) whether in cash or in other forms of future payment promises or by superficial contracts including the abstruse or disguised interest rate.

Rastin Banking is an open source system, and interested readers can access all its detailed technical texts.5

Principles of Rastin Banking

To increase the compliance of banking system with principles and regulations of Islamic religion, and better access to safe financial activity and helping the economy to bloom, fair distribution of possibilities and opportunities, job creation and increasing the welfare of the society, Rastin Banking System was designed and all banks can carry out their activities on this basis. Rastin Banking System is based on special operational, financial, economic, ethical, social, legal, international and organizational principles that based upon the latest scientific achievements of humankind in the field of science and technology with the aim of economic and banking growth and development of the society.

As a brief description, the principles and characteristics of Rastin Banking are based on the following bases and principles:

Operational Principles: By elimination of Riba from banking operations, Rastin Banking forbids superficial application of Islamic contracts and applies them in their real form, and bank acts as an intermediate between depositors and those who receive finance, and bank earns income through offering capital management services and not through interest rates spread. By allocating yield to the money source, the owners of the assets receive their share of profit or loss. All activities will be carried out mechanically and transparently through the internet according to compiled documented regulations. While safeguarding the interests of depositors, the bank is the trustee of the related parties, and the entrepreneur is certain that in the case of loss will not become bankrupt because of insurance coverage safeguards to depositors' deposits. Assessment, supervision, and operation of projects will be based on specialty, responsibility, and independent decision and position of assessor and trustee. The capability and credibility of the entrepreneur will be measured considering his previous tax payments records. The entrepreneur is responsible for his prepared information in his submitted project proposal.

Financial Principles: No bad or delayed loans will be created, and the bank will not bankrupt during the crisis. Because of lower involved risk, bank's capital adequacy rate decreases and bank free reserves increase; and regarding depository characteristic of the bank resources, the legal and precautionary reserves of banks besides central bank will decrease. Depositor, entrepreneur, and the bank will receive their fair share of the yields of sharing. Various financial instruments and banking processes satisfy different society's needs; and by relating Rastin Certificates prices to real sector returns, their prices are stabilized and not only bubble prices emerge, but people with various risk tastes are also attracted and increase the positive interaction between return and risk. Without needing any intermediary, everybody can enter Rastin Certificate Market. Information transparency will make related systems such as tax and social security systems transparent regarding economic activists. Reliable assessment mechanisms have been defined, and reliable supervision background has been provided. Entrepreneur is obliged to observe information disclosure rules relating his project; and by supervision on his operations, corporate governance rules are applied to entrepreneur's company, which leads to the transparency of all information and operations related to the project and asymmetric information and informational rents will be prevented and by applying for insurances, risks are minimized. While facilitating and speeding up the operations, it prevents money laundering and strengthens auditing and internal control of the entrepreneur's company. Online impalpable inspective monitoring prevents bank staff

5 http://www.bidabad.com 
breaches. By separating the accounts, the account of each project and depositor will be kept separately, and the credibility of bank and banking operations increase.

Economic Principles: Rastin Banking decreases capital market deficiencies and contradictions of capital and money markets, and expands and strengthens the insurance sector in the economy. The increased confidence because of bank supervision on entrepreneur and internet accessibility to Rastin Certificate Market provide capital absorption and attraction capability and provides a safe competition environment for entrepreneurs, depositors, transactors and banks with the rivals. New financial instruments diversify financial assets, and by assigning Rastin Certificates to specific projects, goods or assets, paper markets are not generated. The involvement of capital in the project prevents quick movement of assets, which create financial crises because of the liquid flow of funds and by eliminating superficial paper market prevents debt-leverage based crises. Whoever is capable of conducting economic activity can receive financial fund or be employed in job vacancies created by entrepreneurs of the financed projects. Moreover, the application of various financial expertise expands related job opportunities, and all of these provisions improve investment, employment, production, and social welfare and restrict economic and financial fluctuations.

Ethical Principles: Obligation to release real and precise information and documents will persuade honesty and personal peace, and conformity of word and practice, and application of true supervision and decrease of uncertainty and social and individual mental tensions. Forbidding tricks and decrease of financial corruption because of the designed method of assessment, supervision and monitoring systems, and removal of moral hazards potentials lead to put everything in its right place and remove injustice. False devour, incurring a loss to others, betray, gambling and lottery, unfairness, grabby, extortion, waste and squandering, and speculation, conspiracy, and bribery all are forbidden in Rastin Banking and will be fighting against systematically. Observing the rights of weak, implementing financial justice, honoring and respecting rights of people increases mutual consent and benevolence and prevents riba and waste debaucheries openly.

Social Principles: Conducting activities according to pre-described procedures and rules provides the necessary background for regulating the right financial behaviors of people of society and obliges individual discipline. No discrimination, difference, or priority among those with similar characteristics is accepted in Rastin Banking. It teaches people to plan, forecast, and design their financial activities.

Legal Principles: Respecting property rights and individual rights in Rastin Banking is strong. By specifying various aspects of obligations of contracting parties, there will be less reason for juridical settlements; and arbitration facilitates dispute settlement among dissident parties. Moreover, queries of online databases restrict abuses. A new method of execution of enforceable documents in bank loans collection of claims.

International Principles: $7 \times 24 \times 365$ internet accessibility to banking services throughout the world facilitates foreign investment absorption, which provides international mutual interests and political stability background. The virtues of Rastin Banking system introduce it as an operational ideal banking model at the international level, and development of Rastin Certificate Market at international level provides stable assets' anchor. Organizational Principles: Commensurate of the organization to needs, together with proper position appointment and promotion, and generally meritocracy including assignment of qualified persons, organizational communications, duties, responsibilities of personnel and effective punishment and award system are considered and defined in Rastin Banking. Organizational evolution, change, and stability are accompanied by standardization and documentation. Responsiveness of all personnel for their granted authorities prevents the appearance of harmful effects of unofficial organizations and political pressure.

2. Draft of Rastin Banking Bill

"Draft of Rastin Banking Bill" has the following chapters:

Chapter I: Principles and definitions

Chapter 2: Generalities

Chapter 3: Contracts

Chapter 4: Entrepreneur

Chapter 5: Depositor

Chapter 6: Assessment and supervision

Chapter 7: Rastin Certificate

Chapter 8: Rastin Swap Bond (RSB) 
Chapter 9: Rastin Swap Deposit (RSD)

Chapter I0: Rastin Swap Card (RSC)

Chapter II: Rastin Certificate Market (RCM)

Chapter I2: Operation Control and Monitoring System (OCM)

Chapter 13: Collateral Registration System (CRS)

Chapter I4: Mortgage Securitization System (MSS)

Chapter I5: Interbank Withdrawal Protocol (IWP)

Chapter I6: Non-usury Scripless Security Settlement System (NSSSS)

Chapter 17: Rastin Personal Security (RPS)

Chapter 18: Rastin Social Takaful (RST)

Chapter 19: Correcting consumption pattern

Chapter 20: Wasteland reclamation

Chapter 2I: Transparency, information disclosure, and governance

Chapter 22: Enforcement of contents of documents

Chapter 23: Money Laundering Detection system (MLD)

Chapter 24: Safeguarding

Operational Bylaw of Rastin Banking

"Operational Bylaw of Rastin Banking" has the following chapters:

Section One: Rastin Profit and Loss Sharing Base System (PLS)

Chapter I: Definitions and generalities

Chapter 2: Organization

Chapter 3: Professional behavior of assessor and trustee

Chapter 4: Project proposal

Chapter 5: Assessment

Chapter 6: Guarantees, collaterals, and contributions

Chapter 7: Insurance

Chapter 8: Preparing contract

Chapter 9: Supervision

Chapter I0: Entrepreneur

Chapter II: Entrepreneur financial transparency

Chapter I2: Entrepreneur information disclosure

Chapter 13: Entrepreneur governance

Chapter I4: Auditing

Chapter I5: Rastin Sharing Accounting

Chapter 16: Change in timing

Chapter 17: Settlement

Chapter I8: Bank's receipts

Chapter 19: Transform of Subscripted Certificate to Musharakah Certificate

Chapter 20: Commodity inspection and standard

Chapter 2I: Delivery of commodity

Chapter 22: Auxiliary financial instruments

Chapter 23: Force majeure

Chapter 24: Arbitration

Section Two: Financial Subsystems of Rastin PLS banking

Chapter 25: Joalah Financial Sharing (JFS)

Chapter 26: Mudarabah Financial Sharing (MFS)

Chapter 27: Installment Financial Sharing (IFS)

Chapter 28: Rent Financial Sharing (RFS)

Chapter 29: Bail Financial Sharing (BFS) 
Chapter 30: Group Crowd Funding (GCF)

Chapter 3I: Rastin Personal Security (RPS)

Chapter 32: Rastin Social Takaful (RST)

Chapter 33: Sponsor Crowd Funding (SCF)

Chapter 34: Peer to Peer Loan (PPL)

Chapter 35: Rastin Swap Bond (RSB)

Chapter 36: Rastin Swap Deposit (RSD)

Chapter 37: Rastin Swap Card (RSC)

Section Three: Complementary Systems of Rastin Banking

Chapter 38: Rastin Certificate Market (RCM)

Chapter 39: Crowd Funding System (CFS)

Chapter 40: Operation Control and Monitoring System (OCM)

Chapter 4I: Mortgage Securitization System (MSS)

Chapter 42: Collateral Registration System (CRS)

Chapter 43: Serial Commitment Clearance (SCC)

Chapter 44: Interbank Withdrawal Protocol (IWP)

Chapter 45: Non-usury Scripless Security Settlement System (NSSSS)

Chapter 46: Enforcement of the purports of binding bank documents

Chapter 47: Bank's financial transparency, governance and information disclosure

Chapter 48: Money Laundering Detection System (MLD)

\section{Resources of Rastin Banking}

The Persian and English documents of Rastin Banking all are accessible through http://www.bidabad.com in full texts.

\section{Persian Documents}

Persian Books and Detailed Reports

بيزّن بيدآباد، مبانى عرفانى اقتصاد اسلامى، يول، بانك، بيمه و ماليه از ديدكاه حكمت. بزو هشكدة بولى و بانكى، بانكى مركزى إئى جمهورى اسلامى ايران، I383.

http://www.bidabad.com/doc/mabani-erfani-eqtesade-islami.pdf

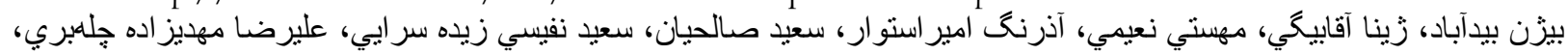

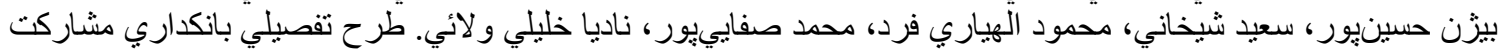
، اداره تحقيقات و برناملريزي، بانك ملي ايران،

http://www.bidabad.com/doc/detailed-pls.pdf

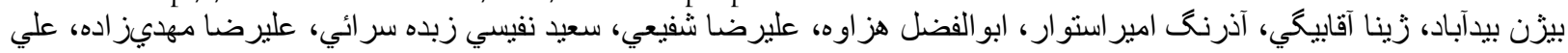

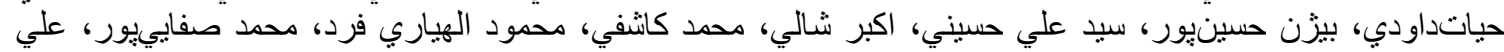

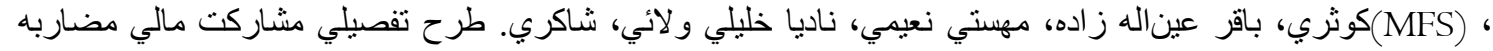

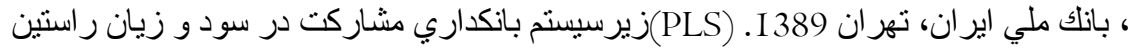

http://www.bidabad.com/doc/detailed-mfs.pdf

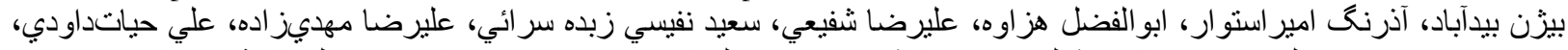

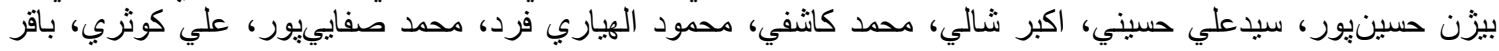

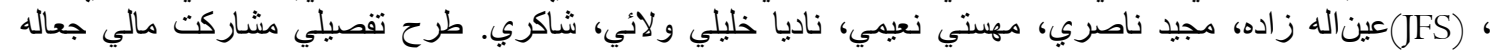

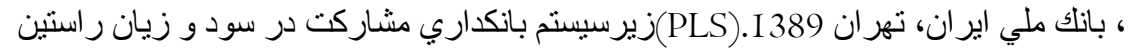

http://www.bidabad.com/doc/detailed-jfs.pdf

بيزن بيدآباد، امير عباس سياهيوش، مهناب ميرز ايیى قاضى، سميه الجبورى، زينب غلامي، امير شريفى، امير شمس، شهر ام اكبرز اده.

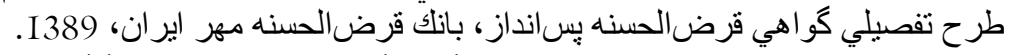

http://www.bidabad.com/doc/gavahi-qarz-tarh-bi-vam.pdf

http://www.bidabad.com/doc/gavahi-qarz-tarh.ppt

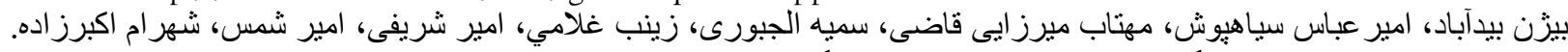

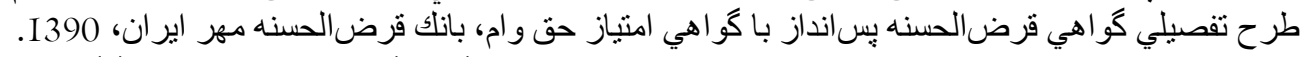
http://www.bidabad.com/doc/gavahi-qarz-tarh-ba-vam.pdf 


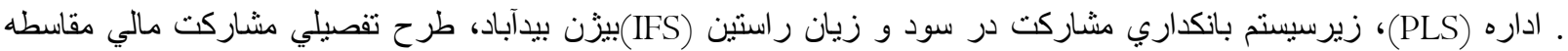

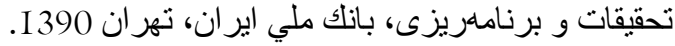
http://www.bidabad.com/doc/detailed-ifs.pdf

ـ اداره تحقيقات (PLS)، زيرسيستم بانكداري مشاركت در سود و زيان راستين (RFS) بيزن بيدآباد، طرح تفصيلي مشاركت مالي اجاره

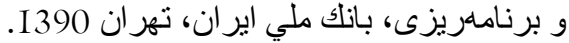
http://www.bidabad.com/doc/detailed-rfs.pdf

، بانك ملي (PLS)، زيرسيستم بانكداري مشاركت در سود و زيان ر استين (RST)بيزن بيدآباد. طر ح تفصيلي تكافل اجتماعى راستين

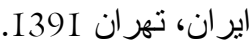

http://www.bidabad.com/doc/detailed-rst.pdf

، بانك ملي اير ان، (PLS)، زيرسيستم بانكداري مشاركت در سود و زيان راستين (BFS)بيزن بيدآباد. طرح تفصيلي تأمين مالى امانى

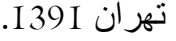
http://www.bidabad.com/doc/detailed-bfs.pdf

، بانك ملي (PLS)، زيرسيستم بانكداري مشاركت در سود و زيان راستين (RPS)بيزن بيدآباد. طرح تفصيلى تأمين شخصى راستين

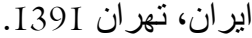
http://www.bidabad.com/doc/detailed-rps.pdf

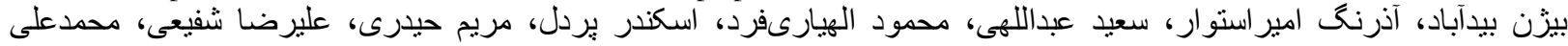

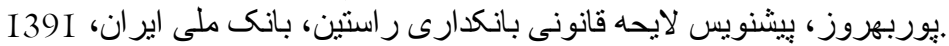
http://www.bidabad.com/doc/rastin-banking-bill.pdf

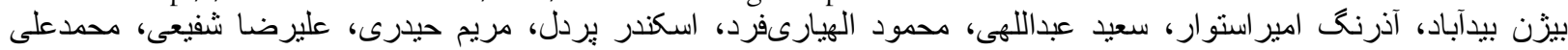

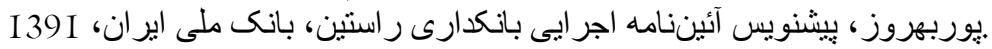
http://www.bidabad.com/doc/rastin-banking-regulation.pdf

)، سيستم مكمل بانكدارى راستين. اداره تحقيقات و برنامعريزى، بانك ملى MLDبيزن بيدآباد، طرح تفصيلى سيستم كثف بولشويى ( ايران. I39I. http://www.bidabad.com/doc/detailed-mld.pdf

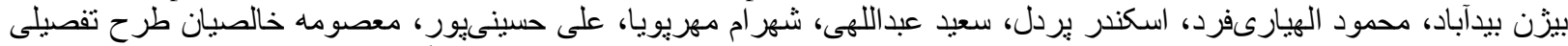

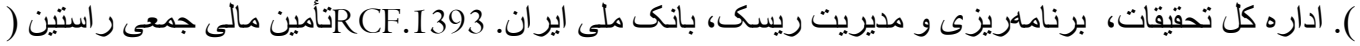
http://www.bidabad.com/doc/detailed-rcf.pdf

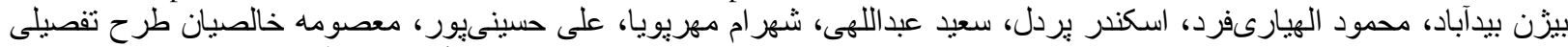

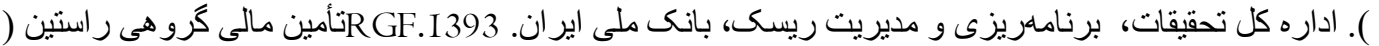
http://www.bidabad.com/doc/detailed-rgf.pdf

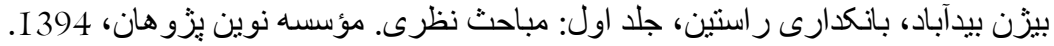
http://www.bidabad.com/doc/rastin-bank-I-fa.pdf http://pub.npdr.ir/product/\%D8\%A8\%D8\%A7\%D9\%86\%DA\%A9\%D8\%AF\%D8\%A7\%D8\%BI \%DB\%8C-\%D8\%BI\%D8\%A7\%D8\%B3\%D8\%AA\%DB\%8C\%D9\%86\%D8\%AC\%D9\%84\%D8\%AF-I /

بيزن بيدآباد، بانكدارى راستين، جلد دوم: مباحث كاربردى. مؤسسه نوين بزّوهان، I394. http://www.bidabad.com/doc/rastin-bank-2-fa.pdf http://pub.npdr.ir/product/\%D8\%A8\%D8\%A7\%D9\%86\%DA\%A9\%D8\%AF\%D8\%A7\%D8\%BI \%DB\%8C-\%D8\%BI\%D8\%A7\%D8\%B3\%D8\%AA\%DB\%8C\%D9\%86-

\%D8\%AC\%D9\%84\%D8\%AF-2-\%D8\%B3\%DB\%8C\%D8\%B3\%D8\%AA\%D9\%85-

\%D9\%86\%D9\%88\%DB\%8C\%D9\%86-

\%D8\%A8\%D8\%A7\%D9\%86\%DA\%A9\%D8\%AF\%D8\%A7/

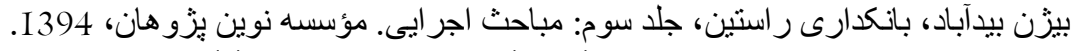
http://www.bidabad.com/doc/rastin-bank-3-fa.pdf http://pub.npdr.ir/product/\%D8\%A8\%D8\%A7\%D9\%86\%DA\%A9\%D8\%AF\%D8\%A7\%D8\%BI \%DB\%8C-\%D8\%BI\%D8\%A7\%D8\%B3\%D8\%AA\%DB\%8C\%D9\%86\%D8\%B3\%DB\%8C\%D8\%B3\%D8\%AA\%D9\%85-\%D9\%86\%D9\%88\%DB\%8C\%D9\%86\%D8\%A8\%D8\%A7\%D9\%86\%DA\%A9\%D8\%AF\%D8\%A7\%D8\%BI\%DB\%8C-\%D8\%A7-2/ 
Persian Papers

هاى فقه متداول در كثف هاى مصرفي و سرمايهكذاري و كاستيبيزن بيدآباد، عبدالرضا هرسيني، تحليل فقهي- اقتصادي ربا در وام

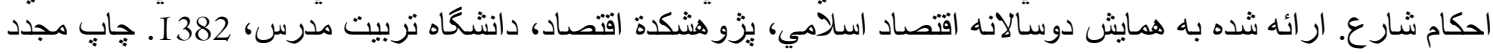

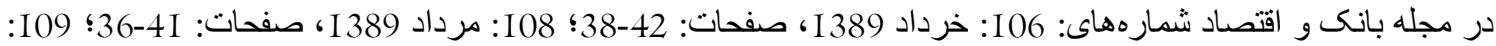

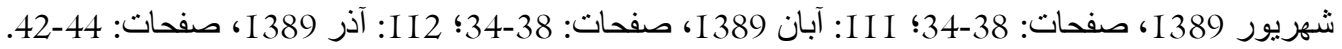
http://www.bidabad.com/doc/reba-fa.pdf

بيزّن بيدآباد، عبدالرضا هرسيني، شركت سهامي بانك غيرربوي و بازبيني ماهيت ربوي و غيرربوي عمليات بانكي منداول. مجموعه

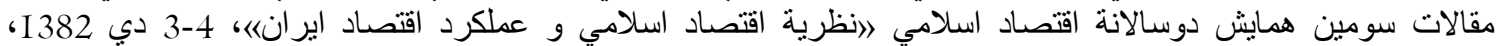

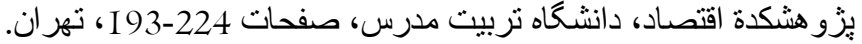

http://www.bidabad.com/doc/sherkat-sahami-bank.pdf http://www.bidabad.com/doc/sherkatbank.ppt

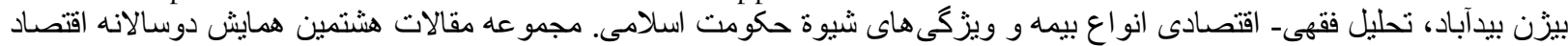

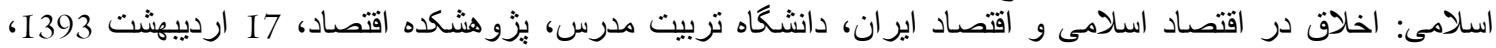
تهران.

http://www.bidabad.com/doc/bimeh.pdf http://www.bidabad.com/doc/bimeh.pptx

بيزّن بيدآباد، تحليل فقهى ـ اقتصادى بيمههاى تأمينى، I389.

http://www.bidabad.com/doc/bimeh-tamini.pdf

بيززن بيدآباد، بيمه و نظرية زنجيرة ورشكستحى، I389. http://www.bidabad.com/doc/bimeh-varshekastegi.pdf

بيزّن بيدآباد، تحليل فقهى ـ اقتصادى بيمه اجبارى، I389. http://www.bidabad.com/doc/bimeh-ejbari.pdf

(بانكداري PLS محود الهياري فرد، فناوري اطلاعات و ارتباطات در تحقق سازوكار مشاركت در سود و زيان ( بيزن بيدآباد،

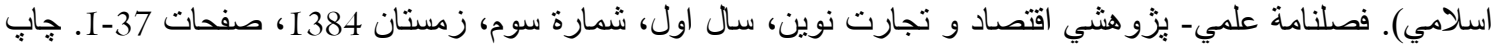

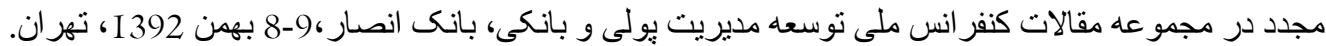
http://prd.moc.gov.ir/jnec/farsi/3rd/Article2.pdf http://www.bidabad.com/doc/Pls_it-fa.pdf

) در بانكداري اسلامي. فصلنامة علمي- بزّو هشي ALMمود الهياري فرد، كار ايیى نسبي مديريت دار ايى و بدهي ( بيزّن بيدآباد،

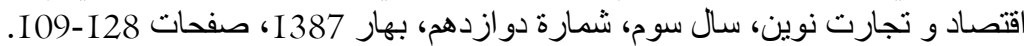

http://www.bidabad.com/doc/alm-farsi.pdf

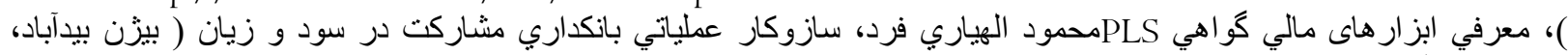

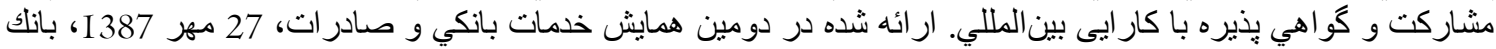

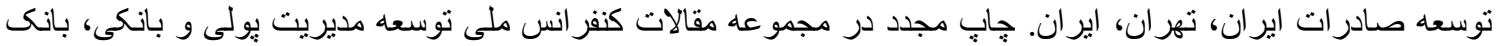
انصار ،96-8 بهمن 13921، تهران.

http://www.bidabad.com/doc/PLS-Banking.pdf

http://www.bidabad.com/doc/PLS-Banking-Export-Deveopment-Bank-2.ppt

http://www.bidabad.com/doc/PLS-Banking-revised.pdf

http://www.bidabad.com/doc/Rastin-Banking-4-revised.pptx

http://www.bidabad.com/doc/pls-tamine-mali.ppt

http://www.bidabad.com/doc/pls-3.ppt

http://www.bidabad.com/doc/pls-mfs-jfs-instructions-93.pptx

http://www.bidabad.com/doc/pls-novin-pajoohan.ppt

http://www.bidabad.com/doc/pls-8-I2-saat.ppt

http://www.bidabad.com/doc/PLS-Banking.pdf

http://www.bidabad.com/doc/PLS-Banking-Export-Deveopment-Bank-2.ppt

محمد صفائي يور، جارجوب باز ار الكترونيكي معاملات كو اهي مشاركت/بذيره در قالب طرح مشاركت در سود و زيان بيزّن بيدآباد،

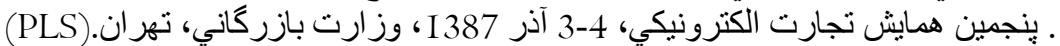

http://www.bidabad.com/doc/charchoobe-bazare-electronic-pls.pdf http://www.ecommerce.gov.ir/EArchive/EArchiveF/Item.asp?ParentID=438ItemID=I82

هاى اقتصادي. مجله بانك و اقتصاد، آذر I388، شماره I03، صفحات 22-27.بانكداري بدون ربا در بر ابر بحران بيزٔن بيدآباد، 
ـ مجمو عه مقالات كنفر انس ملى توسعه مديريت يولى و (PLS) محمود الهياري فرد، حسابداري مشاركت در سود و زيان بيزٔن بيدآباد،

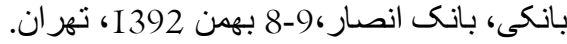

http://www.bidabad.com/doc/PLS-accounting-fa.pdf

بيزن بيدآباد، تنبيث ادوار تجارى با بانكدارى مشاركت در سودو زيان راستين و اقتصاد اخلاق. مجمو عاده مقالات همايش بررسى ابعاد

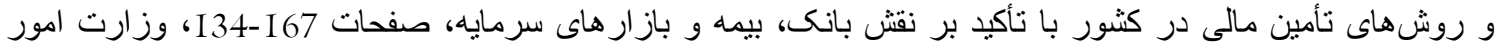

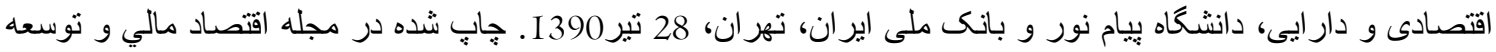

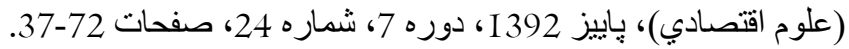

http://fa.journals.sid.ir/ViewPaper.aspx?id=252008 http://www.bidabad.com/doc/pls-business-cycles.pdf http://www.bidabad.com/doc/pls-business-cycles.ppt

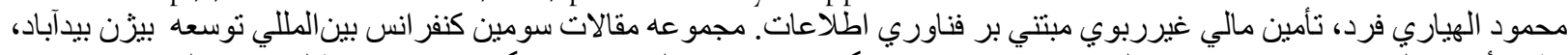

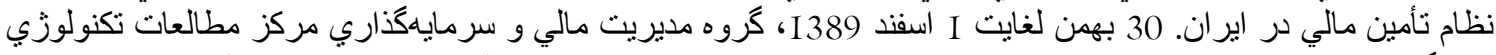

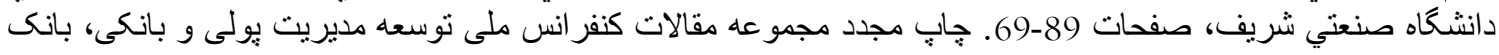

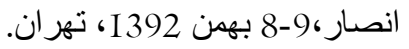

http://www.bidabad.com/doc/non-usury-finance-it-fa.pdf

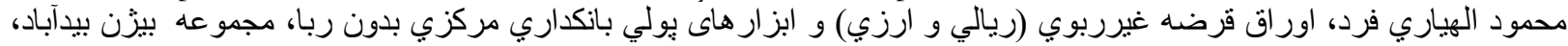

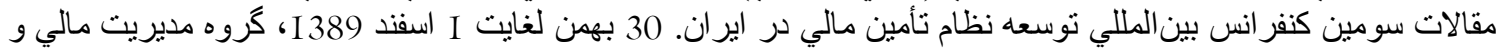

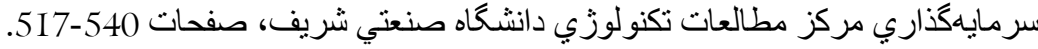
http://www.bidabad.com/doc/Islamic-banking-bond-fa.pdf

) و مشاركت مالي MFS و زيرسيستم هاى مشاركت مالي مضاربه (PLS) (بيزن بيدآباد، نحاهي بر بانكداري مشاركت در سود و زيان (

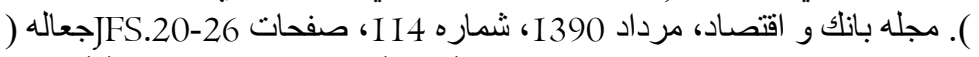

http://www.bidabad.com/doc/negahi-bar-pls-mfs-jfs.pdf

بيزن بيدآباد، بررسي فقهى و حقوقي اوراق قرضه بدون ربا، مجمو عه مقالات همايش ملى بانكدارى اسلامى در تأمين مالى (با تأكيد بر

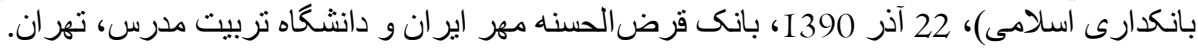

http://www.bidabad.com/doc/legal-analysis-of-non-usury-bonds.pdf http://www.bidabad.com/doc/legal-analysis-of-non-usury-bonds.ppt

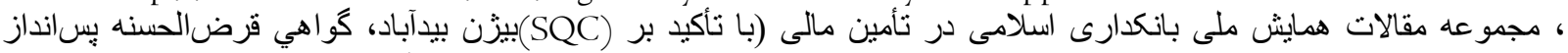

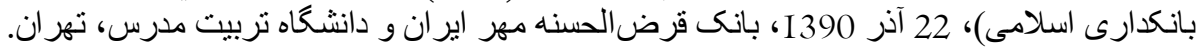

http://www.bidabad.com/doc/gavahi-qarzulhasana-pasandaz.pdf http://www.bidabad.com/doc/gavahi-qarz-paper.ppt

هاى اقتصادي ادواري با ابزار نوين مالي و اصلاح ساختار بانكي، I389.بيزن بيدآباد، جلوكيري از نوسانات و بحران /. http://www.bidabad.com/doc/bohran-va-abzare-mali.pdf

در بانك ملي (PLS)بيزن بيدآباد، مسعود صفرز اده نساجي، زينا آقابيكي، سازمان و تشكيلات بانكداري مشاركت در سود و زيان

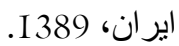
http://www.bidabad.com/doc/PLS-organization.pdf

، 1389. در نوبت جابٍ در يزو هشنامه بانك مسكن، فصلنامه علمى و كاربردى بانك (MFS)بيزّن بيدآباد، مشاركت مالي مضاربه

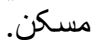

http://www.bidabad.com/doc/mfs-paper-fa.pdf

، يويش، فصلنامه علمى و كاربردى بانك مسكن، سال دوم، شماره ششم، تابستان I392، بيزّن بيدآباد، مشاركت مالي جعاله صفحات 98-I2I. http://www.bidabad.com/doc/jfs-paper-fa.pdf

بيزّن بيدآباد، راهبرد تحول ساختار بانكي، I390. http://www.bidabad.com/doc/rahborde-tahavole-bank.pdf

بيزن بيدآباد، اور اق خز انه بذون ربا (ريالي و ارزي)، 1390. http://www.bidabad.com/doc/interest-free-t-bond-fa.pdf http://www.bidabad.com/doc/interest-free-t-bond-fa.pptx

راستين، بويش، فصلنامه علمى و كاربردى بانك مسكن، سال (PLS)بيزن بيدآباد، باز اريابي تأمين مالي مشاركت در سود و زيان

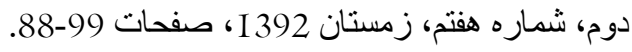


http://www.bidabad.com/doc/pls-marketing.pdf

، I390. در نوبت جاب در يُزو هشنامه بانك مسكن، فصلنامه علمى و كاربردى بانك (IFS)بيزن بيدآباد، مشاركت مالي مقاسطه مسكن.

http://www.bidabad.com/doc/ifs-paper-fa.pdf

بيزن بيدآباد، زبرسيستم هاى مبتنى بر فنآورى اطلاعات و ارتباطات در بانكدارى راستين، مجموعه مقالات همايش ملى خدمات

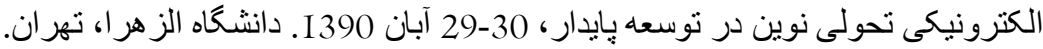

http://www.bidabad.com/doc/Rastin-Bank-IT.pdf

http://www.bidabad.com/doc/Rastin-Bank-IT.ppt

بيزن بيدآباد، امنيت تعاملات بانكى مبتنى بر فناورى اطلاعات در بانكدارى راستين، مجله بانك و اقتصاد شماره: II6: آذر I390 I7 صفحات: 37-40.

http://www.bidabad.com/doc/amniyat-taamolat-banki.pdf

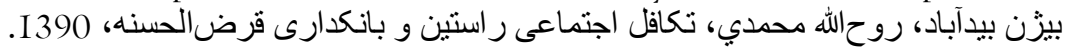

http://www.bidabad.com/doc/takaful-qarzulhasane.pdf

، 1390. در نوبت جاب در يزّو هشنامه بانك مسكن، فصلنامه علمى و كاربردى بانى (RFS)بيزّن بيدآباد، مشاركت مالى اجاره مسكن.

http://www.bidabad.com/doc/rfs-paper-fa.pdf

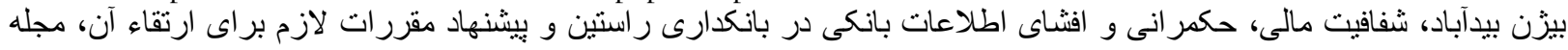

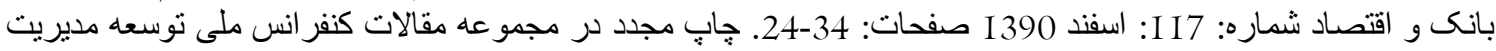

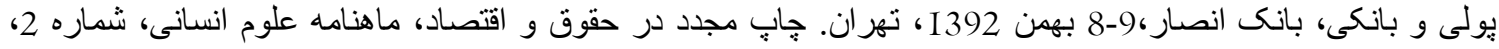

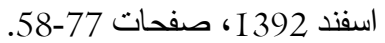

http://www.bidabad.com/doc/shafafiyat.pdf

بيزّن بيدآباد، شفافيت مالى، حاكميت شركتى و افثشاى اطلاعات مجرى (متقاضى منابع مالى) در بانكدارى راستين. تهر ان، I394. http://www.bidabad.com/doc/transparency-entrepreneur-fa.pdf

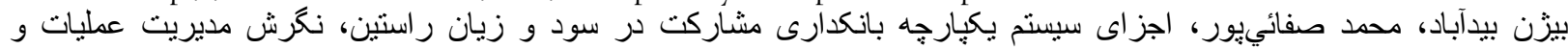

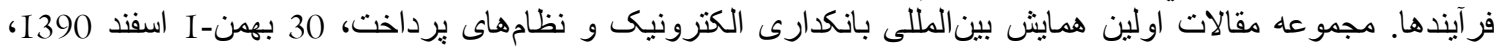

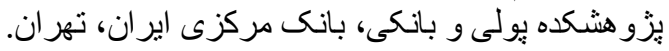

http://www.bidabad.com/doc/ajza-system-rastin.pdf http://www.bidabad.com/doc/ajza-system-rastin.ppt

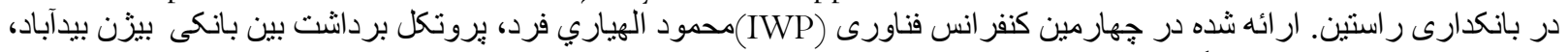

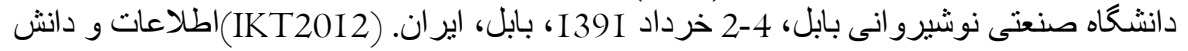

http://www.bidabad.com/doc/iwp-paper-fa.pdf

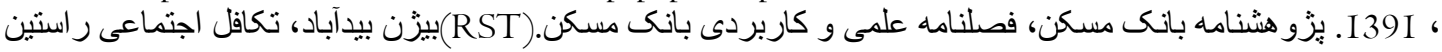
http://www.bidabad.com/doc/rst-paper-fa.pdf

در بانكدارى راستين. I3SI بيزّن بيدآباد. تبديل دار ايى به اور اق بهادار http://www.bidabad.com/doc/mms-paper-fa.pdf

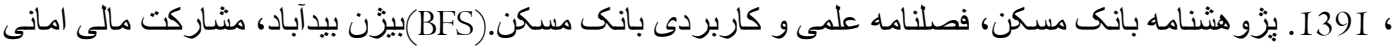
http://www.bidabad.com/doc/bfs-paper-fa.pdf

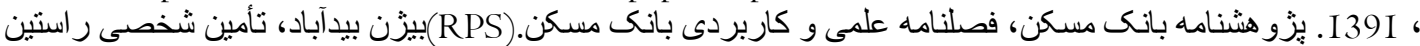
http://www.bidabad.com/doc/rps-paper-fa.pdf

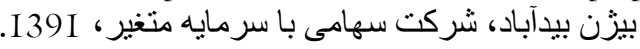

http://www.bidabad.com/doc/vjsc-paper-fa.pdf

بيزن بيدآباد، نحوه جلوكيرى از اسر اف منابع بانكى در بانكدارى راستين

http://www.bidabad.com/doc/esraf-bank-resource-fa.pdf

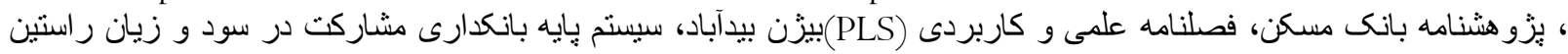

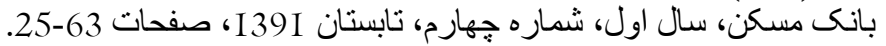

http://www.bidabad.com/doc/pls-base-fa.pdf

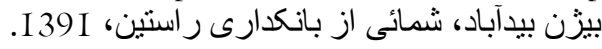
http://www.bidabad.com/doc/rastin-bank-glance-fa.pdf

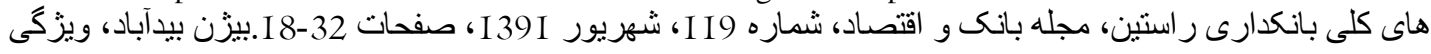


http://www.bidabad.com/doc/rastin-bank-general.pdf http://www.bidabad.com/doc/rastin-bank-general.ppt

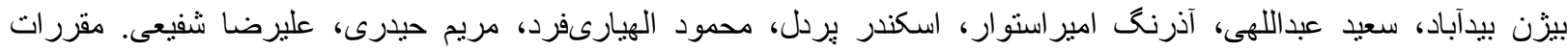

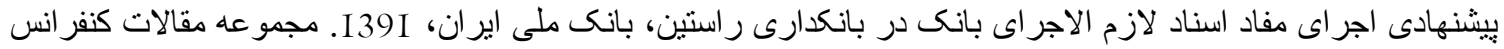
ملى توسعه مديريت بِولى و بانكئ، بانكى، بانكى انصار ،

http://www.bidabad.com/doc/rastin-bank-ejraye-asnad.pdf http://www.bidabad.com/doc/rastin-bank-ejraye-asnad.pptx

بيزن بيدآباد، محمود اللهيارى فرد، بانكدارى راستين و تحول بانكدارى در ايران، نامه اتاق بازركانى، مهر 1392، سال هشتاد و

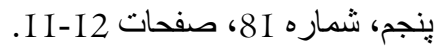
https://www.bidabad.com /doc/rastin-bank-change.pdf https://www.noormags.ir/view/fa/articlepage/I053820

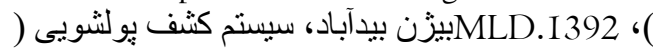

http://www.bidabad.com/doc/mld-paper-fa.pdf

هاى لازمالاجر ا و مكمل بانكدارى راستين بر ایى اصلاح نظام بانكى، اداره تحقيقات و برناماريزي بانك ملي بيزّن بيدآباد، بروزه اير ان، 1392.

http://www.bidabad.com/doc/projects-rastin-banking-fa.pdf

بيزّن بيدآباد، نظرى اجمالى بر بانكدارى ر استين، سيستم نوين بانكدارى اسلامى عملياتى، 1392. http://www.bidabad.com/doc/rastin-bank-bird-eye-view-fa.pdf

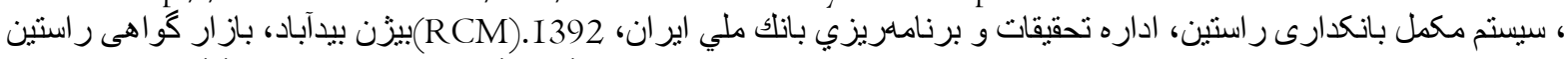
http://www.bidabad.com/doc/rastin-bank-rcm-fa.pdf

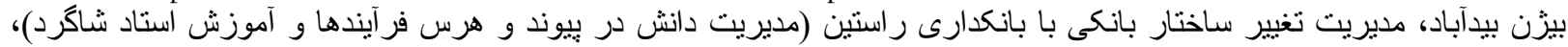

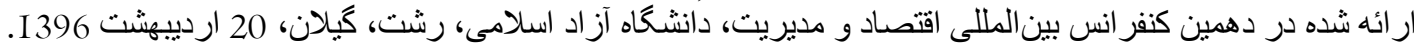
http://www.bidabad.com/doc/change-banking-fa.pdf http://www.bidabad.com/doc/change-banking-fa.pptx

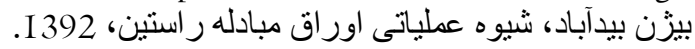
http://www.bidabad.com/doc/rsb-operations-fa.pdf

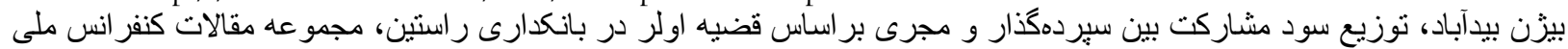

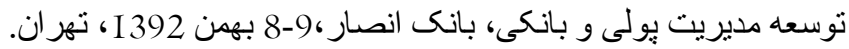
http://www.bidabad.com/doc/profit-distribution-euler-fa.pdf http://www.bidabad.com/doc/profit-distribution-euler-fa.ppt

بيزن بيدآباد، ابز ار هاى مالى مشتقه و قرضه نوين مر بانكدارى راستين، مجمو عه مقالات كنفر انس ملى توسعه مديريت بولى لى و بانكىى،

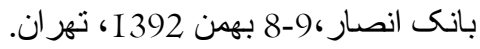

http://www.bidabad.com/doc/rastin-derivatives-fa.pdf

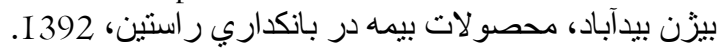
http://www.bidabad.com/doc/rastin-insurance-fa.pdf

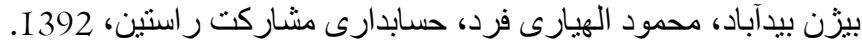
http://www.bidabad.com/doc/rastin-mosharekat-accounting-fa.pdf

بيززن بيدآباد، بانكدارى اخلاقى عملياتى در بانكدارى راستين (اخلاق حرفهاى، حسابرسى، بازرسى، كنترل، بايش و صيانت عمليات)، .1392

http://www.bidabad.com/doc/rastin-ethic-banking-fa.pdf

بيزن بيدآباد، بررسى فقهى و حقوقى مقررات بانكدارى بدون ربا در ايران و بِيشنهاد لايحها و وآئينامه اجر ائى بانكدارى راستين.

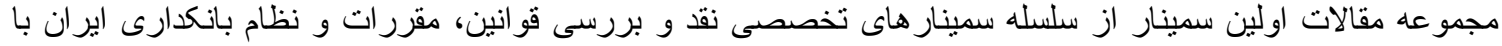

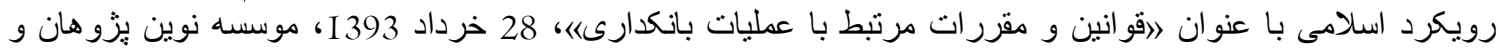

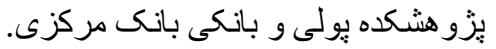
http://www.bidabad.com/doc/jurist-legal-banking-fa.pdf

، مجمو عه مقالات جهار مين همايش سالانه بانكدارى الكترونيكى و نظامهاى بِرداخت، (RCF)بيزّن بيدآباد، تأمين مالى جمعى راستين 6-7 بهمن 1393.

http://www.bidabad.com/doc/rcf-paper-fa.pdf http://www.bidabad.com/doc/rcf-paper-fa.pptx 
(بيزّن بيدآباد، تأمين مالى كرو هى راستين RGF.I393. http://www.bidabad.com/doc/rgf-paper-fa.pdf

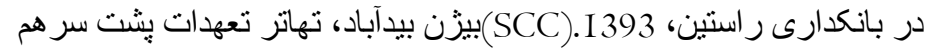
http://www.bidabad.com/doc/scc-paper-fa.pdf

بيزن بيدآباد، سيرده مبادله راستين (RSD). 1393 ، http://www.bidabad.com/doc/rsd-paper-fa.pdf

بيزن بيدآباد، كارت مبادله راستين (RSC).I393 ، http://www.bidabad.com/doc/rsc-paper-fa.pdf

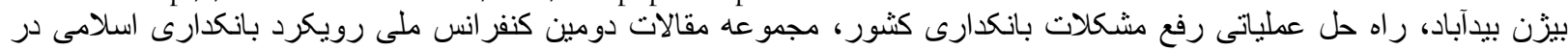

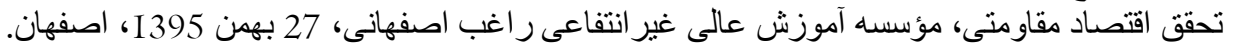
http://www.bidabad.com/doc/Iran-Banking-Reform-fa.pdf http://www.bidabad.com/doc/Iran-Banking-Reform-fa.pptx

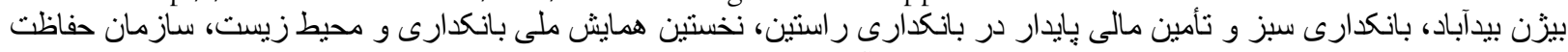

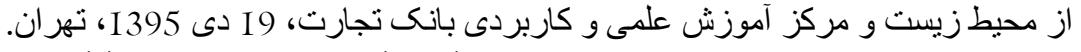

http://www.bidabad.com/doc/green-rastin-banking-fa.pdf http://www.bidabad.com/doc/green-rastin-banking-fa.ppt

محمود الهيارى فرد، بيزن بيدآباد، حسابدارى اقلام زير خط ترازنامه در حسابدارى مشاركت راستين، 1395، تهران. http://www.bidabad.com/doc/off-balance-rastin-accounting-fa.pdf

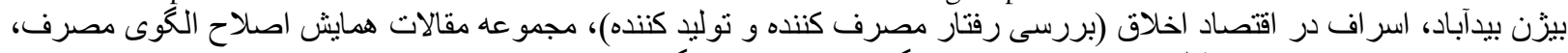

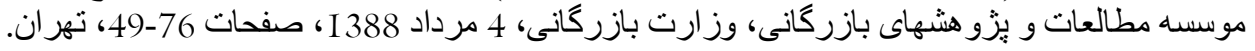
http://www.bidabad.com/doc/esraf-eghtesade-akhlagh.pdf

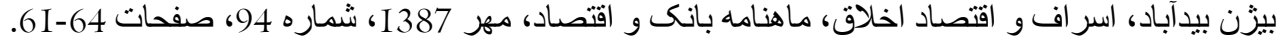

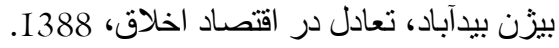
http://www.bidabad.com/doc/taadol-eghtesade-akhlagh.pdf

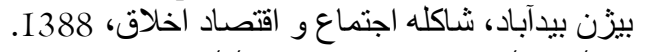
http://www.bidabad.com/doc/shakeleh-ejtema-eghtesade-akhlagh.pdf

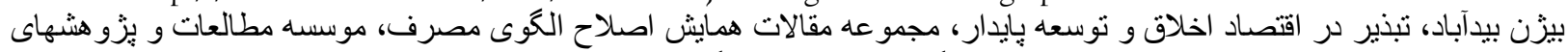

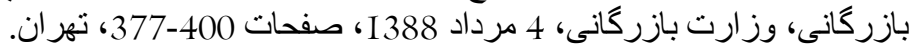

http://www.bidabad.com/doc/toseeh-payedar-eghtesade-akhlagh.pdf

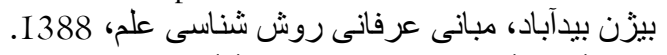
http://www.bidabad.com/doc/mabani-ravesh-shenasi-elm.pdf

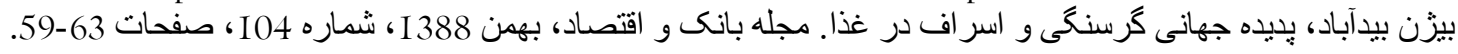
http://www.bidabad.com/doc/gorosnegi/padideh-gorosnegi.pdf

بيزن بيدآباد، تعادل در اقتصاد اخلاق و اقتصساد نئوكلاسيك، 1389. http://www.bidabad.com/doc/taadol-akhlaq-neoclassic.pdf

\section{English Documents}

English Books

Bidabad, Bijan, New Operational Islamic Banking System, Volume One, Theoretical Foundations, Lap Lambert Academic Publishing, OmniScriptum GmbH \& Co. KG, 20I4, ISBN: 978-3-659-54463-7. http://www.amazon.com/Rastin-Banking-Operational-Theoretical-

Foundations/dp/3659544639/ref=sr_I_2?s=books\&ie=UTF8\&qid $=\mathrm{I} 4025635 \mathrm{I} 8 \&$ sr $=\mathrm{I}-2$ http://pub.npdr.ir/product/rastin-banking/

Bidabad, Bijan, New Operational Islamic Banking System, Volume Two, Applicational Issues, Lap Lambert Academic Publishing, OmniScriptum GmbH \& Co. KG, 20I4, ISBN: 978-3-659-552I0-6. http://www.amazon.com/Rastin-Banking-II-OperationalApplication/dp/3659552100/ref=sr_I_I?s=books\&ie=UTF8\&qid=I404643353\&sr=I -I http://pub.npdr.ir/product/rastin-banking-volume-ii/ 
Bidabad, Bijan, New Operational Islamic Banking System, Volume Three, Legal Issues, Lap Lambert Academic Publishing, OmniScriptum GmbH \& Co. KG, 202I.

\section{English Papers}

Bidabad, Bijan, Economic-juristic analysis of usury in consumption and investment loans and contemporary jurisprudence shortages in exploring legislator commandments. International Journal of Islamic Business \& Management, 3(2), I-I5, 2019.

https://www.cribfb.com/journal/index.php/ijibm/article/view/275 http://www.bidabad.com/doc/reba-en.pdf

Bidabad, Bijan, Non-Usury Bank Corporation (NUBankCo), The Solution to Islamic banking. International Journal of Shari'ah and Corporate Governance Research, 2(I), 53-66, 2019.

https://www.cribfb.com/journal/index.php/ijscgr/article/view/276 http://www.bidabad.com/doc/NUBankCo-en.pdf

Bidabad, Bijan, Mahmoud Allahyarifard. IT role in fulfillment of Profit \& Loss Sharing (PLS) mechanism. International Journal of Islamic Banking and Finance Research, 3(2), 44-59, 2019.

https://www.cribfb.com/journal/index.php/ijibfr/article/view/274

http://www.bidabad.com/doc/english-pls-5.pdf

http://www.bidabad.com/doc/pls-it-en.ppt

Bidabad, Bijan, Mahmoud Allahyarifard. Implementing IT to Fulfill Profit \& Loss Sharing Mechanism. Islamic Finance News (IFN), Vol. 3, Issue 3, 6, February 2006, pp. II-I5.

http://www.bidabad.com/doc/summery-pls-it-I.pdf

Bidabad, Bijan; Mahmoud Allahyarifard. Assets and Liabilities Management in Islamic Banking. International Journal of Islamic Banking and Finance Research, 3(2), 32-43, 2019.

https://www.cribfb.com/journal/index.php/ijibfr/article/view/272 http://www.bidabad.com/doc/alm-english.pdf

Bidabad, Bijan. Non-Usury Banking Fits the Change Strategy: The Solution to Revive the Economy. American Economic \& Social Review, 5(I), 49-6I, 2019.

https://www.cribfb.com/journal/index.php/aesr/article/view/283

http://www.bidabad.com/doc/PLS-paper-en-5.pdf

Bidabad, Bijan, Mahmoud Allahyarifard. The Executive Mechanism of Rastin Profit and Loss Sharing (PLS) Banking. Indian Journal of Finance and Banking, 3(I), 23-39, 2019.

https://www.cribfb.com/journal/index.php/ijfb/article/view/308

http://www.bidabad.com/doc/PLS-banking-Executive-Mechanism.pdf

http://www.bidabad.com/doc/pls-en.pptx

Bidabad, Bijan, Mahmoud Allahyarifard. IT-Based Usury-Free Financial Innovations. American Finance \& Banking Review, 4(I), 39-49, 2019 .

https://www.cribfb.com/journal/index.php/amfbr/article/view/289

http://www.bidabad.com/doc/non-usury-finance-it-en.pdf

Bidabad, Bijan, Mahmoud Allahyarifard. Usury-Free Bonds and Islamic Central Banking Monetary Instruments. 2010.

http://www.bidabad.com/doc/Islamic-banking-bond-en.pdf

Bidabad, Bijan. Stabilizing Business Cycles by PLS Banking and Ethic Economics. International Journal of Shariah and Corporate Governance Research, 2(I), 67-82, 2019.

https://www.cribfb.com/journal/index.php/ijscgr/article/view/284

http://www.bidabad.com/doc/pls-business-cycles-en.pdf

Bidabad, Bijan, Mahmoud Allahyarifard. Accounting Procedures for Profit and Loss Sharing (PLS) Banking. 2010. http://www.bidabad.com/doc/PLS-accounting-en.pdf

Bidabad, Bijan, Abul Hassan, Ben Ali Mohamed Sami, Mahmoud Allahyarifard. Interest-Free Bonds and Central Banking Monetary Instruments. International Journal of Economics and Finance. Vol. 3, no. 3, Aug 20I I, pp.234-24I. 
http://www.ccsenet.org/journal/index.php/ijef/article/download/I I665/8300

Bidabad, Bijan, Fluctuations and Business Cycles Prevention by New Financial Instruments and Banking Structure Reform. International Journal of Accounting \& Finance Review, 4(I), 35-50, 2019. https://www.cribfb.com/journal/index.php/ijafr/article/view/282 http://www.bidabad.com/doc/Fluctuations-and-Cycles.pdf

Bidabad, Bijan, Mudarebah Financial Sharing (MFS). Journal of Islamic Economics, Banking and Finance, JIEBF, Volume - I0, Number - I, January - April 20I4, pp. 56-68. http://www.bidabad.com/doc/mfs-paper-en.pdf http://ibtra.com/pdf/journal/vI0_nI_article3.pdf

Bidabad, Bijan, Joalah Financial Sharing (JFS). Journal of Islamic Economics, Banking and Finance, Volume-I2, No. I, January-March, 2016, pp. 33-48. http://www.bidabad.com/doc/jfs-paper-en.pdf http://ibtra.com/pdf/journal/vI2_nI_article2.pdf

Bidabad, Bijan, Interest-Free Treasury Bonds (IFTB). International Journal of Shari'ah and Corporate Governance Research, 2(2), 13-2I, 2019. https://www.cribfb.com/journal/index.php/ijscgr/article/view/306 http://www.bidabad.com/doc/interest-free-t-bond-en.pdf http://www.bidabad.com/doc/iftb-en.pptx

Bidabad, Bijan, Interest-Free Treasury Bonds (IFTB), Islamic Finance and Legal Clarifications. International Joumal of Islamic Business \& Management, 3(I), 2I-29, 2019.

http://www.bidabad.com/doc/interest-free-t-bond-feqhi-en.pdf https://www.cribfb.com/journal/index.php/ijibm/article/view/258/353

Bidabad, Bijan, Abul Hassan, Ben Ali Mohamed Sami, Mahmoud Allahyarifard. Interest-Free Bonds Financial Innovation, A Monetary Instrument for Economy at Crisis. Journal of Economic Cooperation and Development (JECD). 32, I, 20I I, 55-70.

http://www.sesric.org/jecd/jecd_articles/ARTI0I0220I-2.pdf

Bidabad, Bijan, Deposits and Loans Interest Rates Lag Structure and Business Cycles (Case Study of United States). 20II.

http://www.bidabad.com/doc/Interest-Rates-Lags-and-Cycles.pdf

Bidabad, Bijan, Installment Financial Sharing (IFS): A Financial Subsystem of Rastin PLS Banking. International Journal of Islamic Banking and Finance Research, 3(I), 28-42, 2019. https://www.cribfb.com/journal/index.php/ijibfr/article/view/267 http://www.bidabad.com/doc/ifs-paper-en.pdf

Bidabad, Bijan, Abul Hassan, Does The Interest Rate Form Business Cycle?. International Journal of Accounting \& Finance Review, 4(I), 29-34, 2019. https://www.cribfb.com/journal/index.php/ijafr/article/view/28I http://www.bidabad.com/doc/interest-rate-cycle.pdf

Bidabad, Bijan, Rent Financial Sharing (RFS). Journal of Islamic Economics, Banking and Finance, Vol. I0 No. 2, pp.: 38-53, April-June 20I4.. http://www.bidabad.com/doc/rfs-paper-en.pdf http://ibtra.com/pdf/journal/vI0_n2_article2.pdf

Bidabad, Bijan, Rastin Social Takaful (RST). Joumal of Islamic Economics, Banking and Finance, JIEBF, Volume II, Number - I, January - March 2015, pp.: 13-23. http://ibtra.com/pdf/journal/vII_nI_articleI.pdf http://www.bidabad.com/doc/rst-paper-en.pdf

Bidabad, Bijan, Mortgage Securitization System (MSS), A Complementary System of Rastin Banking. International Journal of Law and Management (IJLMA), Vol. 59 Issue: 6, pp.778-783, 2017. Emerald Group Publishing Limited https://doi.org/I0.I I08/IJLMA-05-2016-0045 http://www.bidabad.com/doc/mss-paper-en.pdf 
Bidabad, Bijan, Bail Financial Sharing (BFS): A Financial Subsystem of Rastin PLS Banking. International Journal of Islamic Banking and Finance Research, 3(I), 2I-27, 2019.

https://www.cribfb.com/journal/index.php/ijibfr/article/view/266

http://www.bidabad.com/doc/bfs-paper-en.pdf

Bidabad, Bijan, Rastin Personal Security (RPS). Journal of Islamic Economics, Banking and Finance, JIEBF, Volume - II, Number - 2, April - June 2015, pp. 47-6I.

http://ibtra.com/pdf/journal/vII_n2_article3.pdf

http://www.bidabad.com/doc/rps-paper-en.pdf

Bidabad, Bijan, Joint Stock Company with Variable Capital (JSCVC), International Journal of Law and Management (IJLMA), Emerald Group Publishing Limited, Vol. 56, Iss: 4, pp.302 - 3I0, 2014.

http://dx.doi.org/I0.II08/IJLMA-09-20I2-003I

http://www.bidabad.com/doc/vjsc-paper-en.pdf

Bidabad, Bijan, Rastin Profit and Loss Sharing (PLS) Base System. Journal of Islamic Economics, Banking and Finance, pp. 32-57, Vol. 9, No. 4, Oct-Dec 2013. http://ibtra.com/pdf/journal/v9_n4_article2.pdf http://www.bidabad.com/doc/pls-base-en.pdf

Bidabad, Bijan, Money Laundering Detection System (MLD), A Complementary System of Rastin Banking, Journal of Money Laundering Control, Vol. 20 Issue: 4, pp. 354-366, 2017. https://doi.org/I0.I I08/JMLC-04-2016-0016 http://www.bidabad.com/doc/mld-paper-en.pdf

Bidabad, Bijan, General Characteristics of Rastin Banking. Asian Finance \& Banking Review, 3(2), 7-25, 2019. https://www.cribfb.com/journal/index.php/asfbr/article/view/35I http://www.bidabad.com/doc/rastin-bank-general-en.pdf http://www.bidabad.com/doc/rastin-bank-general-en.ppt

Bidabad, Bijan, A glance at Rastin Banking, 2019. http://www.bidabad.com/doc/rastin-bank-glance-en.pdf

Bidabad, Bijan, Rastin Banking, New Operational Islamic Banking System (A bird's eye view). Islamic Finance News (IFN), Vol. I0, Issue: 28, I7, July, 20I3, pp. I6-I8.

http://www.bidabad.com/doc/rastin-bank-bird-eye-view-en.pdf

Bidabad, Bijan, Islamic Monetary Policy. International Journal of Islamic Banking and Finance Research, 3(2), I-I6, 2019.

https://www.cribfb.com/journal/index.php/ijibfr/article/view/269 http://www.bidabad.com/doc/islamic-monetary-policy-en.pdf

Bidabad, Bijan, Rastin Certificate Market (RCM), Complementary System of Rastin Banking. International Journal of Islamic Business \& Management, 3(I), 35-43, 2019.

https://www.cribfb.com/journal/index.php/ijibm/article/view/260 http://www.bidabad.com/doc/rastin-bank-rcm-en.pdf

Bidabad, Bijan, Sovereign Wealth Fund Asset and Liability Management by Rastin Banking Financial Instruments (Rastin Certificates and Rastin Swap Bonds), American Finance \& Banking Review, 4(I), I-I6, 2019. https://www.cribfb.com/journal/index.php/amfbr/article/view/285 http://www.bidabad.com/doc/swf-alm-en.pdf http://en.ndf.ir/international-conference/nic-2013/conference-full-paper.aspx

Bidabad, Bijan, Mahmoud Allahyarifard, Interbank Withdrawal Protocol (IWP), Complementary System of Rastin Banking. International Journal of Islamic Business \& Management, 3(I), 30-34, 2019. https://www.cribfb.com/journal/index.php/ijibm/article/view/259 http://www.bidabad.com/doc/iwp-paper-en.pdf

Bidabad, Bijan, Roohollah Mohammadi; Mahshid Sherafati, Social Takaful and Qard ul-Hassanah Banking Convergences (A Functional Approach), Journal of Applied Science and Agriculture (JASA) November issue 2013.

http://www.bidabad.com/doc/takaful-en.pdf 
Bidabad, Bijan, Change Management of Banking System at National Level by Rastin Banking (Knowledge Management, Empowerment, Prune and Graft, and Apprenticeship), Review of Behavioral Aspect in Organizations and Society, 2019, I(I), 57-70 .

https://doi.org/I0.32770/rbaos.volI57-70

http://www.bidabad.com/doc/change-banking-en.pdf

http://www.bidabad.com/doc/change-banking-en.pptx

Bidabad, Bijan, Insurance Products in Rastin Profit and Loss Sharing Banking. Indian Journal of Finance and Banking, 3(I), 40-54, 2019.

https://www.cribfb.com/journal/index.php/ijfb/article/view/344

http://www.bidabad.com/doc/rastin-insurance-en.pdf

Bidabad, Bijan, Abul Hassan, Dynamic Lag Structure of Deposits and Loans Interest Rates and Business Cycles Formation. Proceeding of the 3rd International Conference on Economics, Political, Law and Fiscal Sciences (EPLS 'I4), World Scientific and Engineering Academy and Society (WSEAS). Transilvania University of Brasov, Brasov, Romania, June 26-28, 2014.

http://www.bidabad.com/doc/interest-rates-cycle-en.pdf http://www.bidabad.com/doc/interest-rates-cycle-en.ppt

Bidabad, Bijan, Rastin Crowdfunding (RCF): A Financial Subsystem of Rastin Banking. International Journal of Islamic Banking and Finance Research, 3(I), I3-20, 2019.

https://www.cribfb.com/journal/index.php/ijibfr/article/view/265

http://www.bidabad.com/doc/rcf-paper-en.pdf

Bidabad, Bijan, Rastin Group Funding (RGF): A Financial Subsystem of Rastin Banking. International Journal of Islamic Banking and Finance Research, 3(I), 43-48, 2019.

https://www.cribfb.com/journal/index.php/ijibfr/article/view/268

http://www.bidabad.com/doc/rgf-paper-en.pdf

Bidabad, Bijan, Mahshid Sherafati, Bank Information Disclosure, Financial Transparency and Corporate Governance in Rastin Banking, International Journal of Shariah and Corporate Governance Research, 2(I), I-I3, 2019.

https://www.cribfb.com/journal/index.php/ijscgr/article/view/257

http://www.bidabad.com/doc/shafafiyat-en.pdf

Bidabad, Bijan, Mahshid Sherafati, Financial Transparency, Governance and Public Disclosure for Entrepreneur (Financial Resource Receiver) in Rastin Banking System. Tehran, Iran, 2015. http://www.bidabad.com/doc/transparency-entrepreneur-en.pdf

Bidabad, Bijan, Azarang Amirostovar, Mahshid Sherafati, Financial Transparency, Corporate Governance and Information Disclosure of the Entrepreneur's Corporation in Rastin Banking. International Journal of Law and Management (IJLMA), Emerald Group Publishing Limited, Vol:59, Iss:5, pp.636-65I, 2017. https://doi.org/IO.I I08/IJLMA-0I-2016-0003

Bidabad, Bijan, Saeid Abdollahi, Mahshid Sherafati, Rohollah Mohammadi, Proposed Regulations for Enforcement of Purports of Binding Banking Documents in Rastin Banking. International Journal of Small and Medium Enterprises, 2(I), 23-49, 2019.

https://www.cribfb.com/journal/index.php/ijsmes/article/view/32I

http://www.bidabad.com/doc/rastin-bank-ejraye-asnad-en.pdf

Bidabad, Bijan, Saeed Abdollahi, Mahshid Sherafati, Enforcement of the Purports of Binding Banking Documents in Rastin Banking - Part I. International Journal of Law and Management (IJLMA), Emerald Group Publishing Limited, Vol:59, Iss:I, pp. 52-65, 2017. https://doi.org/I0.I I08/IJLMA-07-20I5-004I

Bidabad, Bijan, Saeed Abdollahi, Mahshid Sherafati, Enforcement of the Purports of Binding Banking Documents in Rastin Banking - Part II. International Journal of Law and Management (IJLMA), Emerald Group Publishing Limited, Vol:59, Iss:2, pp.178-191, 2017.

https://doi.org/I0.I 108/IJLMA-I0-2015-0055 
Bidabad, Bijan, Mahshid Sherafati, Sustainable Financing and Anti-Squandering Measures in Rastin Banking. International Journal of Law and Management (IJLMA), Emerald Group Publishing Limited, Vol: 59, Issue: 6, pp. 939-949, 2017. https://doi.org/I0.I 108/IJLMA-04-2016-0037 http://www.bidabad.com/doc/esraf-bank-resource-en.pdf

Bidabad, Bijan, Serial Commitments Clearance (SCC) in Rastin Banking. International Journal of Law and Management (IJLMA), Vol. 57, Iss: 6, 2015, pp. 600-609, Emerald Group Publishing Limited. http://dx.doi.org/I0.I108/IJLMA-02-2015-0007 http://www.bidabad.com/doc/scc-paper-en.pdf

Bidabad, Bijan, Rastin Swap Deposit (RSD): A Financial Account of Rastin Banking. International Journal of Islamic Banking and Finance Research, 3(2), 17-23, 2019.

https://www.cribfb.com/journal/index.php/ijibfr/article/view/270 http://www.bidabad.com/doc/rsd-paper-en.pdf

Bidabad, Bijan, Rastin Swap Card (RSC): A Financial Instrument of Rastin Banking. International Journal of Islamic Banking and Finance Research, 3(2), 24-31, 2019.

https://www.cribfb.com/journal/index.php/ijibfr/article/view/27I http://www.bidabad.com/doc/rsc-paper-en.pdf

Bidabad, Bijan, Mahshid Sherafati, Operational Ethical Banking in Rastin Banking (Professional Ethics, Audit, Inspection, Control, Monitoring and Preservation). International Journal of Law and Management (IJLMA), Emerald Group Publishing Limited, Vol: 58, Iss: 4, 2016, pp. 4I6-443. http://dx.doi.org/I0.II08/IJLMA-07-2015-0037 http://www.bidabad.com/doc/rastin-ethic-banking-en.pdf

Bidabad, Bijan, Abul Hassan, Dynamic Lag Structure of Deposits and Loans Interest Rates and Business Cycles Formation. Journal of Financial Regulation and Compliance, Vol. 25 Issue: 2, pp.I I4-I32, 2017. http://dx.doi.org/I0.II08/JFRC-09-20I6-0078

Bidabad, Bijan, General Regulatory Framework in Rastin Profit and Loss Sharing Banking (Part I-Operational Context). Journal of Business and Finance in Emerging Markets, JBFEM, [S.1.], v. I, n. I, p. II-26, May 20I8. ISSN 2580-5568. https://doi.org/I0.32770/jbfem.volI II-26 http://www.bidabad.com/doc/rastin-regulatory-en-I.pdf

Bidabad, Bijan, General Regulatory Framework in Rastin Profit and Loss Sharing Banking (Part II-Legal Groundwork). Journal of Business and Finance in Emerging Markets, JBFEM, JBFEM, [S.1.], v. I, n. 2, p. I09-I26, nov. 20I8. ISSN 2580-5568.

https://doi.org/I0.32770/jbfem.volI I09-I26 http://www.bidabad.com/doc/rastin-regulatory-en-II.pdf

Bidabad, Bijan, General Regulatory Framework in Rastin Profit and Loss Sharing Banking (Part III-Auxiliary Provisions). Journal of Business and Finance in Emerging Markets, JBFEM, May 2019, Vol 2, No. I, pp. 5I-65. ISSN 2580-5568. https://doi.org/I0.32770/jbfem.vol25I-66 http://www.bidabad.com/doc/rastin-regulatory-en-III.pdf

Bidabad, Bijan, Rohollah Mohammadi, Mahshid Sherafati, Organizational Design and Rules in Rastin Profit and Loss Sharing Banking. International Journal of Small and Medium Enterprises, 2(I), 9-22, 2019. https://www.cribfb.com/journal/index.php/ijsmes/article/view/320 http://www.bidabad.com/Organizational-structure-paper-en.pdf

Bidabad, Bijan, Mahmoud Allahyarifard, Mahshid Sherafati. Rastin Partnership Accounting, Part I: General Procedure, Journal of Islamic Accounting and Business Research, 2019. http://www.bidabad.com/doc/rastin-partnership-accounting-I-en.pdf https://doi.org/I0.I I08/JIABR-04-2016-0049

Bidabad, Bijan. Rastin Partnership Accounting, Part II: Mudarabah Financial Sharing (MFS). 2019. http://www.bidabad.com/doc/rastin-partnership-accounting-II-en.pdf

Bidabad, Bijan. Rastin Partnership Accounting: Part III: Instalment Financial Sharing (IFS). 2019. 
http://www.bidabad.com/doc/rastin-partnership-accounting-III-en.pdf

Bijan Bidabad, Squandering in Ethic Economics: Consumer and Producer Behaviors Analysis. Squandering in Ethic Economics: Consumer and Producer Behaviors Analysis. International Journal of Islamic Business \& Management, 3(2), 30-4I. 2019.

https://www.cribfb.com/journal/index.php/ijibm/article/view/279

http://www.bidabad.com/doc/esraf-eghtesade-akhlagh-en.pdf

Bijan Bidabad, Overconsumption in Ethic Economics and Sustainable Development. International Journal of Islamic Business \& Management, 3(2), 42-5I, 2019.

https://www.cribfb.com/journal/index.php/ijibm/article/view/280

http://www.bidabad.com/doc/toseeh-payedar-eghtesade-akhlagh-en.pdf

Bijan Bidabad, Alarm to Global Hunger Phenomenon and Food Squandering. American International Journal of Social Science Research, 4(I), 3I-34, 2019.

https://www.cribfb.com/journal/index.php/aijssr/article/view/307

http://www.bidabad.com/doc/Global-hunger-en.pdf

Bijan Bidabad, Equilibrium in ethic economics. International Journal of Islamic Business \& Management, 3(2), I623. 2019. https://www.cribfb.com/journal/index.php/ijibm/article/view/277

http://www.bidabad.com/doc/taadol-eghtesade-akhlagh-en.pdf

Bijan Bidabad, Mahshid Sherafati, Disposition (Shakilah) of Society and Ethic Economics. Argos journal, 2016, pp. I8I-I92. http://nebula.wsimg.com/8bfebcdI33a57I2fff54I8e5367d27a0?AccessKeyId=C26FF9F9DF562879I B69\&disposition $=0 \&$ alloworigin $=\mathrm{I}$ http://www.bidabad.com/doc/shakeleh-ejtema-eghtesade-akhlagh-en.pdf

Bijan Bidabad, Ethic Economics, Fair Economy, New Economy, Sustainability and other Related Disciplines. International Journal of Islamic Business \& Management, 3(2), 24-29, 2019. https://www.cribfb.com/journal/index.php/ijibm/article/view/278 http://www.bidabad.com/doc/fair-economy-en.pdf

\section{Copyrights}

Copyright for this article is retained by the author(s), with first publication rights granted to the journal. This is an open-access article distributed under the terms and conditions of the Creative Commons Attribution license (http://creativecommons.org/licenses/by/4.0/). 